\title{
Conservative Management of Invasive Placentation: Two Cases with Different Surgical Approaches
}

\author{
Emily E. Fay, MD ${ }^{1}$ Barbara Norquist, $\mathrm{MD}^{2} \quad$ Jennifer Jolley, $\mathrm{MD}^{3}$ Melissa Hardesty, MD, MPH
}

1 Department of Obstetrics and Gynecology, University of Washington School of Medicine, Seattle, Washington

2 Division of Gynecologic Oncology, Department of Obstetrics and Gynecology, University of Washington School of Medicine,

Seattle, Washington

${ }^{3}$ Division of Maternal-Fetal Medicine, Department of Obstetrics and Gynecology, University of Washington School of Medicine,

Seattle, Washington

${ }^{4}$ Division of Gynecologic Oncology, Alaska Women's Cancer Care,

Anchorage, Alaska

Am J Perinatol Rep 2016;6:e212-e215.
Address for correspondence Emily Fay, MD, Department of Obstetrics and Gynecology, University of Washington School of Medicine, Box 356460, 1959 NE Pacific St., Seattle, WA 98195 (e-mail: efay@uw.edu).

\author{
Abstract \\ Keywords \\ - placenta accreta \\ - invasive placenta \\ - cesarean \\ hysterectomy \\ - robotic surgery
}

Background When placenta accreta complicates a delivery, the typical management is to perform a cesarean hysterectomy. Other management strategies, including leaving the placenta in situ, have been attempted and supported in some cases. This may allow for an interval hysterectomy, which can potentially decrease average blood loss and/or allow a minimally invasive approach to the hysterectomy.

Cases We present two cases of women with invasive placentation managed conservatively with interval hysterectomy. One woman was managed with robotic-assisted laparoscopic surgery and the other with an open surgical approach.

Conclusion These cases highlight the successful use of conservative management for invasive placentation in two stable patients and showcase the novel use of a roboticassisted laparoscopic surgery for management of invasive placentation.
Placenta accreta is the abnormal attachment of the placenta to the myometrium and is rising as the incidence of cesarean delivery is increasing. When placenta accreta complicates a delivery, the historical recommendation is to perform a cesarean hysterectomy. ${ }^{1}$ Peripartum hysterectomy carries significant risks including injury to the genitourinary tract and substantial blood loss. Other management options include leaving the placenta in place and closing the cesarean hysterotomy incision, then utilizing pelvic arterial embolization or balloon occlusion catheters to decrease blood flow and encourage spontaneous resorption of the placenta with or without subsequent methotrexate therapy. This may allow uterine conservation in patients for whom future fertility is desired, or may allow for an interval hysterectomy, which can potentially decrease average blood loss and/or allow a minimally invasive approach to the hysterectomy.

received

February 27, 2016

accepted after revision

April 11, 2016
DOI http://dx.doi.org/

$10.1055 / \mathrm{s}-0036-1584232$. ISSN 2157-6998.
We present two cases in which conservative management was used at the time of delivery with both women undergoing an interval hysterectomy, one of whom was managed with robotic-assisted laparoscopic surgery and the other patient requiring an open surgical approach.

\section{Case 1}

A 30-year-old gravida 2, para 1, with a history of one prior cesarean delivery, presented at 34 weeks 1 day of gestation for management of suspected placenta accreta in the setting of maternal religious beliefs precluding the receipt of blood products. Prenatal ultrasound showed a complete placenta previa with myometrial thinning where the placenta was seen just posterior to the bladder with increased vascularity in the bladder wall.

Copyright $\odot 2016$ by Thieme Medical Publishers, Inc., 333 Seventh Avenue, New York, NY 10001, USA. Tel: +1(212) 584-4662.
License terms

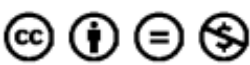


The patient underwent a repeat classical cesarean section through a Pfannenstiel incision with bilateral tubal ligation at 35 weeks 0 day of gestation. She delivered a female infant weighing 2,340 g with Apgar scores of 6 and 8. The placenta was adherent to the uterus and bulging vessels crossed the lower uterine segment but with no exterior invasive placental tissue. The bladder was adherent to the most inferior part of the uterus without placental invasion into the bladder. With these findings and concern for hemorrhage if either placental removal or hysterectomy was attempted, the decision was made to leave the placenta in situ. The umbilical cord was tied off with 0 Vicryl (Ethicon US, Cincinnati, $\mathrm{OH}$ ) and replaced into the uterus. The uterine incision was repaired in a single layer with looped 0 Maxon (Covidien, Minneapolis, MN). Total blood loss was $800 \mathrm{~mL}$.

In the postoperative period, she received prophylactic antibiotics and was monitored for signs of bleeding and infection. She remained in the hospital for 8 days postdelivery and then returned home with plan for close follow-up until placental resorption. The patient was seen every 2 weeks. At 8 weeks postpartum, she developed unmanageable pelvic pain related to uterine distention, thought to be hematometra due to cervical obstruction from the placenta. Her enlarged uterus also caused urinary retention and the decision was made to proceed with hysterectomy. She underwent embolization of the uterine vessels and then a robotic-assisted laparoscopic hysterectomy and bilateral salpingectomy. Port placement was as follows: five port sites, a midline camera port placed $25 \mathrm{~cm}$ above the pubic symphysis, a right upper quadrant assist port, and $38 \mathrm{~mm}$ robotic ports. Findings included an edematous bladder with neovascularity that was densely adherent to the anterior surface of the uterus. The placenta was bulging in the lower uterine segment and there was an area on the anterior uterus where near full-thickness involvement of the myometrium was apparent. The blood loss for the case was $500 \mathrm{~mL}$, much of which was secondary to back bleeding on the uterus due to difficulty coagulating the uterine vessels on the side where the placenta was located. After detachment from the vagina, the uterus and cervix were removed through the vagina without morcellation.

Pathologic evaluation recorded a uterus and placenta weighing $536 \mathrm{~g}$, with the uterus measuring $10.4 \times 8.5 \times 6.5 \mathrm{~cm}$ and the attached placenta measuring $15.5 \times 9.5 \times 3.0 \mathrm{~cm}$. The placenta had invaded through the myometrium but was confined by the serosa of the uterus, consistent with placenta increta. The patient recovered well without complication and was discharged home on the second postoperative day.

\section{Case 2}

A 33-year-old gravida 3, para 2 at 26 weeks 2 days of gestation presented following her second episode of vaginal bleeding. Her pregnancy was complicated by placenta previa in the setting of two prior cesarean deliveries, with concern for placenta accreta. She received betamethasone and magnesium sulfate for transport. After arrival at our institution, the patient developed acute vaginal bleeding estimated at $1 \mathrm{~L}$ and the decision was made to move forward with delivery.
A repeat classical cesarean delivery was performed through a midline abdominal incision, with delivery of a male infant weighing 1,088 $\mathrm{g}$ with Apgar scores of 2, 3, and 7. Excessive bleeding was encountered despite avoidance of the placenta. The hysterotomy was quickly closed to decrease bleeding. Examination showed blood vessels emanating from the placenta traversing the uterus to the anterior abdominal wall. Multiple large vessels coursed through the lower uterine segment, which had been replaced by invasive placenta. The bladder flap could not be appreciated due to the complete obliteration of the anterior wall of the uterus. The bulging placenta and lower uterine segment extended to the sidewalls of the pelvis. Total blood loss was $4,000 \mathrm{~mL}$ at this point, but with closure of the hysterotomy, there was no further bleeding. Given the already large hemorrhage and extensive invasion of the placenta into the uterus and pelvis, the decision was made to leave the placenta in situ and not attempt additional surgery at that time. The patient received six units of packed red blood cells during her hospital course.

Postoperatively, she underwent bilateral uterine artery embolization with Gelfoam (Pfizer Injectables, Kalamazoo, $\mathrm{MI}$ ), and additional Gelfoam embolization of the anterior division internal iliac arterial branches that supplied the uterus and placenta percreta. She received prophylactic antibiotics and was monitored for signs of bleeding and infection. She remained in the hospital for 5 days postdelivery and was then discharged to local housing. She was followed weekly with ultrasounds to assess for interval resorption of placental tissue. Due to the ongoing possibility of hemorrhage or infection with the placenta left in situ, a plan was made for interval hysterectomy.

The patient underwent a second uterine artery embolization at 7 weeks postpartum. At 8 weeks postpartum, she underwent a cystoscopy with ureteral stent placement, followed by exploratory laparotomy, hysterectomy, and resection of the dome of the bladder with cystotomy repair. On cystoscopy, there was extensive vascularity seen in the posterior bladder. During surgery, the entire anterior uterus was replaced by the placenta with blood vessels traveling from the placenta into the anterior abdominal wall and extensive involvement of the dome of the bladder with vascularity and placental invasion, necessitating resection of a portion of the dome of the bladder. Estimated blood loss for the case was $2,600 \mathrm{~mL}$, and she received four units of packed red blood cells and two units of fresh frozen plasma intraoperatively.

Pathology showed a uterus weighing $854 \mathrm{~g}$, measuring $21.1 \times 13.5 \times 6.5 \mathrm{~cm}$. The endometrial cavity was filled with blood and autolytic placenta measuring approximately $12 \times 10 \times 4.5 \mathrm{~cm}$, which invaded the full thickness of the endomyometrium (-Fig. 1).

The patient was discharged home on postoperative day 5 with a Foley catheter. She had a cystourethrogram on postoperative day 8 , which showed a large bladder leak; therefore, the catheter was kept in place. She was subsequently managed by an urologist and ultimately required 6 weeks of Foley catheter drainage to allow the bladder to heal. 

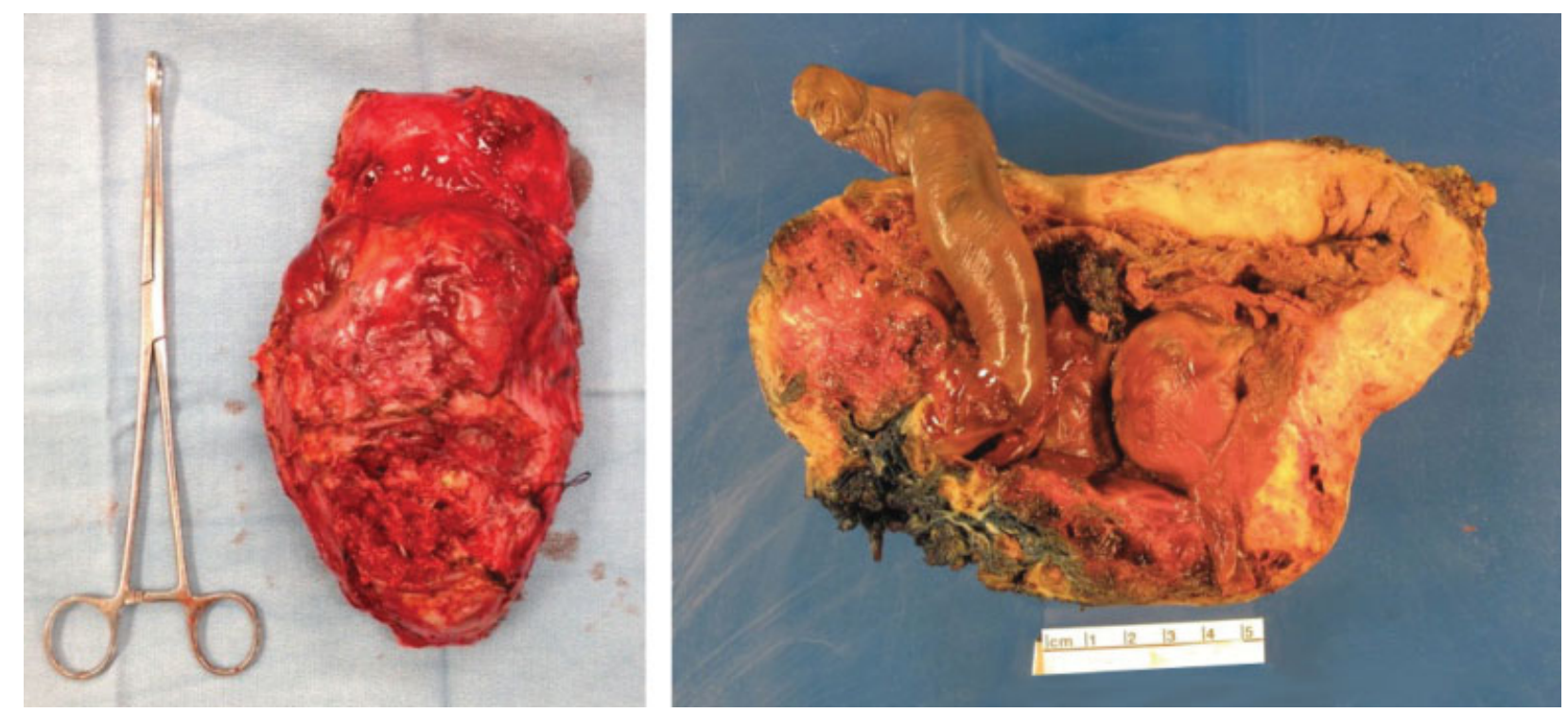

Fig. 1 Photo on left shows uterus following postpartum hysterectomy, with ring forceps for scale. Photo on right shows uterus following postpartum hysterectomy, opened to reveal retained placenta.

\section{Discussion}

Although cesarean hysterectomy is the most common management for invasive placentation in the United States, another option for a stable patient includes conservative management with placental retention and uterine conservation at the time of delivery. This may allow uterine conservation in patients for whom future fertility is desired, or may allow for an interval hysterectomy, which can potentially avoid the possible complications of cesarean hysterectomy, including injury to the bladder and/or ureters, massive hemorrhage, and even maternal death and/or allow a minimally invasive approach to the hysterectomy. In cases of placenta percreta, this approach may be used to try to minimize the amount of bladder or other organ tissue that requires resection. Lee et al described two cases of conservative management with planned interval hysterectomy in two women with placenta percreta with bladder invasion. ${ }^{2}$ Both women underwent cesarean delivery with the placenta left in situ, received pelvic artery embolization, and methotrexate therapy postpartum, and then underwent a delayed hysterectomy with partial resection of the bladder.

Studies that have evaluated conservative treatment in which the placenta is left in situ without planned hysterectomy have found that this approach can have significant risks. A retrospective, multicenter study of 167 women treated conservatively for placenta accreta reported subsequent hysterectomy rates of $22 \%$, and severe maternal morbidity was found in 10 cases, including 3 women who developed a deep vein thrombosis or pulmonary embolism, 7 women who developed sepsis, and 1 maternal death related to intraumbilical cord administration of methotrexate. $^{3}$ A smaller retrospective review included 26 patients who underwent conservative management of placenta accreta. ${ }^{4}$ In this group, the subsequent hysterectomy rate was $19 \%$. In addition, two of the women developed complications secondary to postoperative uterine artery embolization, including necrosis of the uterus and bladder and development of ischemia of the leg necessitating a thrombectomy. In a review of management of placenta percreta, the authors included 36 women who were treated conservatively. ${ }^{5}$ In this group, $58 \%$ of the women underwent a subsequent hysterectomy. In addition, $44 \%$ suffered postoperative hemorrhage and $25 \%$ developed infections, compared with only $12 \%$ who developed postoperative hemorrhage and $12 \%$ who developed infections in those who had primary hysterectomy or local resection.

It is known that nonobstetric hysterectomies performed via a minimally invasive approach, compared with open hysterectomies, offer many advantages including quicker return to normal activities, lower intraoperative blood loss, shorter hospital stay, fewer wound or abdominal wall infections, and smaller incisions. ${ }^{6}$ It can be reasoned that this would be similar for obstetric hysterectomies. For conservative management of placenta accreta, only a few case reports have been published detailing a minimally invasive approach to hysterectomy, with only one case report using robotic technology. ${ }^{7-11}$

Both of the patients presented in these cases had invasive placentation diagnosed before delivery, were managed at a tertiary care center with a multidisciplinary approach, and were stable at the time of cesarean delivery, which allowed them to be managed conservatively. In addition, both patients were able to return for close outpatient follow-up. In each case, the patient ultimately had a hysterectomy.

In the first case, we chose conservative management given the patient's religious beliefs precluding the receipt of blood products to attempt to decrease her overall blood loss. She had a cesarean delivery with minimal blood loss. She was then managed with prophylactic antibiotics, one postpartum ultrasound, and followed by twice-monthly visits. She ultimately developed hematometra, pain, and urinary retention necessitating a hysterectomy, but was able to undergo uterine artery embolization followed by an uncomplicated robotic-assisted laparoscopic hysterectomy, potentially allowing for less blood loss and shorter recovery time. 
In the second case, we chose conservative management because the patient had substantial placental invasion into the bladder and anterior abdominal wall and already had a large blood loss at the time of delivery, and the extensive surgery that would have been required to resect the remaining placenta percreta would have been life threatening. The patient underwent immediate postoperative uterine artery embolization. She received prophylactic antibiotics and was followed up with weekly ultrasounds. She then underwent a second uterine artery embolization before her open hysterectomy and bladder resection. By delaying her hysterectomy and utilizing uterine artery embolization, theoretically the patient was able to undergo the hysterectomy and bladder resection with less morbidity than would have occurred if performed at the time of cesarean delivery already complicated by massive hemorrhage.

These cases support the consideration of conservative management for an invasive placenta in a stable patient, particularly in cases in which peripartum hysterectomy could lead to significant morbidity, and the use of a minimally invasive approach when feasible. Conservative management should be performed with caution, however, as it does carry its own significant risks, and there are still many unknowns with this approach, including what constitutes appropriate monitoring and follow-up.

\section{Acknowledgments}

The authors would like to thank the two women discussed in this case report for allowing them to share their stories.

\section{References}

1 Committee on Obstetric Practice. Committee opinion no. 529: placenta accreta. Obstet Gynecol 2012;120(1):207-211

2 Lee PS, Bakelaar R, Fitpatrick CB, Ellestad SC, Havrilesky LJ, Alvarez Secord A. Medical and surgical treatment of placenta percreta to optimize bladder preservation. Obstet Gynecol 2008;112(2 Pt 2): 421-424

3 Sentilhes L, Ambroselli C, Kayem G, et al. Maternal outcome after conservative treatment of placenta accreta. Obstet Gynecol 2010; 115(3):526-534

4 Bretelle F, Courbière B, Mazouni C, et al. Management of placenta accreta: morbidity and outcome. Eur J Obstet Gynecol Reprod Biol 2007;133(1):34-39

5 Clausen C, Lönn L, Langhoff-Roos J. Management of placenta percreta: a review of published cases. Acta Obstet Gynecol Scand 2014;93(2):138-143

6 Nieboer TE, Johnson N, Lethaby A, et al. Surgical approach to hysterectomy for benign gynaecological disease. Cochrane Database Syst Rev 2009;8(3):CD003677

7 Arendas K, Lortie KJ, Singh SS. Delayed laparoscopic management of placenta increta. J Obstet Gynaecol Can 2012;34(2):186-189

8 Boes S, Mahdi H, Khoury F, Kebria MM. Peripartum roboticassisted laparoscopic hysterectomy after second-trimester pregnancy loss with placenta increta. Obstet Gynecol 2013;122(2 Pt 2): 440-444

9 Garibaldi S, Perutelli A, Baldacci C, Gargini A, Basile S, Salerno MG. Laparoscopic approach for peripartum hysterectomy. J Minim Invasive Gynecol 2013;20(1):112-114

10 Ochalski ME, Broach A, Lee T. Laparoscopic management of placenta percreta. J Minim Invasive Gynecol 2010;17(1): $128-130$

11 Skinner BD, Golichowski AM, Raff GJ. Laparoscopic-assisted vaginal hysterectomy in a patient with placenta percreta. JSLS 2012; 16(1):143-147 